\title{
High-sensitivity light-addressable potentiometric sensors using silicon on sapphire functionalised with self-assembled organic monolayers
}

Jian Wang, Yinglin Zhou' ${ }^{1}$, Michael Watkinson², Julien Gautrot, Steffi Krause*

School of Engineering and Materials Science, Queen Mary University of London, Mile End Road, London E1 4NS, UK

${ }^{1}$ School of Chemistry and Molecular Engineering, Peking University, Beijing, 100871, China

${ }^{2}$ School of Biological and Chemical Sciences, Queen Mary University of London, Mile End Road, London E1 4NS, UK

*Corresponding author: s.krause@qmul.ac.uk, Telephone +44 (0)2078823747

\begin{abstract}
:
The use of self-assembled organic monolayers as the insulator in Light-Addressable Potentiometric Sensors (LAPS) is shown for the first time. Silicon on sapphire (SOS) substrates were modified with 1-octadecene or undecylenic acid. Experiments with a photoresist pattern as the model system showed the same good spatial resolution that was previously obtained with a conventional insulator on SOS, but also a significant improvement in the photocurrent contrast and therefore the sensitivity of LAPS. Surface potential imaging was validated by studying micropatterns of poly(allylamine hydrochloride) (PAH) and DNA on a PAH template using LAPS. The results showed that self-assembled organic monolayers provide an excellent, reproducible, ultrathin insulator for LAPS, which can be readily functionalised and used for high-sensitivity LAPS imaging and sensing.
\end{abstract}

Keywords: Light-Addressable Potentiometric Sensors, Scanning Photo-induced Impedance Microscopy, self-assembled monolayers, undecylenic acid

\section{Introduction}

Light-addressable potentiometric sensors (LAPS) can record local changes in the surface potential, thus determining a particular analyte under test with spatial resolution [1-5]. In LAPS, different spots on an electrolyte/insulator/silicon (EIS) field-effect structure are addressed by scanning a focused light beam across the sample thereby exciting local photocurrents [6]. The potential changes are measured as a shift of the depletion region of the photocurrent voltage curve along the voltage axis. We have shown that the same measurement technology can also be used to measure the local impedance by recording the maximum photocurrent in inversion [7] and termed this technique Scanning Photo-induced Impedance Microscopy (SPIM).

Recently, LAPS has been used successfully for the measurement of extracellular action potentials [8];[9] and for the measurement of extracellular $\mathrm{pH}$ changes [10]. All the above LAPS measurements suffered from poor, ill-defined resolution of about 10-100 $\mu$ m, thereby making subcellular imaging impossible. Using silicon on sapphire (SOS) substrates and a two-photon effect, we have achieved submicrometer resolution [11]. This progress in resolution could contribute to the development of a platform for imaging the ion channel activity and metabolic behaviour of a single cell using LAPS.

The silicon-electrolyte interface has been extensively studied by electrochemical characterization. The dark current measured at $p$-type silicon at cathodic potentials is very small $\left(<2-3 \mu \mathrm{A} / \mathrm{cm}^{2}\right)[12]$ and the silicon-electrolyte junction can work itself as a barrier. However, under illumination, significant cathodic currents have been observed [12-14]. 
Hence an additional barrier is required for LAPS devices to function in electrolyte solutions. Traditionally, insulators typically used in the semiconductor industry, such as thermally grown silicon oxide and silicon nitride have been employed [6] [15].

The sensitivity of LAPS has been improved by using a thin anodic oxide as the insulator [11]. In this work, we sought to advance the technique further through the replacement of the insulator with an ultrathin organic monolayer. Self-assembled monolayers (SAMs), which are directly grafted onto silicon without an intervening layer of silicon dioxide, are stable and can be prepared with mild and highly efficient methods [16]. Their excellent insulating effects have been demonstrated in electrochemical impedance measurements [17]. The selective control of surface chemistry and the small thickness are expected to extend the applications of field effect devices in the fields of atomic scale device fabrication, diverse molecular device development [18] and chemical and biological sensors [19-22]. A carboxyl terminated silicon surface can be directly obtained without need for a protection step as olefinic carboxylic acids react primarily through the alkene moiety [22-24]. In this work two different monolayers were produced with 1-octadecene or undecylenic acid and were used as an insulator in photocurrent measurements.

\section{Materials and methods}

\subsection{Materials}

Silicon on sapphire (SOS) (Monocrystal, Russia) with a $0.5 \mu$ m thick silicon (100) layer (boron doped, $0.05 \Omega \mathrm{cm}$ ) and a $1 \mu \mathrm{m}$ thick silicon (100) layer (boron doped, $0.1 \Omega \mathrm{cm}$ ) on a $475 \mu \mathrm{m}$ thick sapphire substrate was used. The cleaning $\left(\mathrm{H}_{2} \mathrm{O}_{2}, 30 \% ; \mathrm{H}_{2} \mathrm{SO}_{4}, 96 \%\right.$; acetic acid, 100\%) and etching (HF, 48\%) reagents were of VLSI grade and supplied by SigmaAldrich, UK. A Sylgard 184 poly (dimethyl siloxane) (PDMS) kit was purchased from Dow Corning. Tetrahydrofuran and dichloromethane (HPLC grade), poly(allylamine hydrochloride) $(\mathrm{PAH}, 70 \mathrm{kDa})$, rhodamine B isothiocyanate (RBITC), 1-octadecene (>95.0\%) and undecylenic acid (98-100.5\%) were obtained from Sigma-Aldrich, UK. The photoresist SU-8 2000.5 and EC solvent were purchased from Chestech Ltd., UK. The oligonucleotide sequences were purchased from Shanghai Sangon Biotechnology Co. Ltd. (Shanghai, China) and have the following sequences: AAG TCC GTG GTA GGG CAG GTT GGG GTG ACT (TBA), AGT CAC CCC AAC CTG CCC TAC CAC GGA CT (rTBA). GeneFinder ${ }^{\mathrm{TM}}$ was obtained from Zeesan Biotech (China) to label the double stranded DNA (dsDNA). $10 \mathrm{mM}$ phosphate buffer $\mathrm{pH} 7.4$ was used to dissolve the DNA and dye. The $\mathrm{pH}$ sensitivity was measured using buffers with the following compositions: $\mathrm{pH} 3-7.5$, citric acid/ sodium phosphate dibasic; pH 8.5, trizma base; $\mathrm{pH}$ 9.4, carbonate/bicarbonate. For other photocurrent measurements and leakage current measurement, $10 \mathrm{mM}$ tris buffer solution at $\mathrm{pH} 7.4$ was used. All the buffers contained $137 \mathrm{mM} \mathrm{NaCl}$ and $2.7 \mathrm{mM} \mathrm{KCl}$. High-purity water $(18 \mathrm{M} \Omega \mathrm{cm})$ was used to prepare all solutions.

\subsection{Sensor preparation}

A silicon on sapphire (SOS) wafer was cut into $7 \mathrm{~mm} \times 7 \mathrm{~mm}$ pieces. In order to form the ohmic contact, the chips were etched in 10\% HF, $30 \mathrm{~nm} \mathrm{Cr}$ and $150 \mathrm{~nm}$ Au were thermally evaporated onto one corner of the chip using an Edwards Coating System E306A and the samples were then heated to $350^{\circ} \mathrm{C}$ for $5 \mathrm{~min}$. The SOS substrate was cleaned in a hot piranha solution $\left(3: 1 \mathrm{H}_{2} \mathrm{SO}_{4}(96 \%) / \mathrm{H}_{2} \mathrm{O}_{2}(30 \%)\right)$ at $100^{\circ} \mathrm{C}$ for 15 min and then rinsed 
copiously with ultrapure (Milli-Q) water. The H-terminated silicon surface was obtained by chemical etching in 10\% HF for 5 min. During this time, undecylenic acid was deoxygenated under argon in a Schlenk tube at $90^{\circ} \mathrm{C}$ for $30 \mathrm{~min}$ and then cooled to room temperature under continuous bubbling of argon. The freshly prepared $\mathrm{H}$-Si substrate was transferred into the Schlenk tube with continuous argon bubbling for $30 \mathrm{~min}$. Grafting was performed overnight at $160^{\circ} \mathrm{C}$ adapting a procedure described in [25]. The functionalized surface was then rinsed in hot acetic acid at $75^{\circ} \mathrm{C}$ and copious amounts of water. Scheme 1a shows the schedule of the monolayer modification process. For the reaction of Si surface with 1octadecene, a temperature of $180^{\circ} \mathrm{C}$ was used. After the reaction, the samples were rinsed successively with tetrahydrofuran and dichloromethane and blown dry with nitrogen.

X-ray photoelectron spectroscopy (XPS) experiments were performed on the undecylenic acid monolayer surface at the nanoLAB of Newcastle University, UK, using a Kratos Axis Nova spectrometer, with the CasaXPS software. Survey scans were carried out over the 1100 0 eV range with a $1.0 \mathrm{eV}$ step size, a $100 \mathrm{~ms}$ dwell time, and an analyzer pass energy of $100 \mathrm{eV}$. High-resolution scans were run with a $0.1 \mathrm{eV}$ step size, a dwell time of $100 \mathrm{~ms}$, and the analyser pass energy set to $20 \mathrm{eV}$. The scan regions were Si2p (97-107 eV), C1s (278-294 eV), and O1s (526-542 eV).

\subsection{Pattern preparation for resolution measurements}

To measure the lateral resolution in the semiconductor materials used, a clearly defined edge on the surface had to be fabricated [11]. For that purpose, films of SU-8 with sharp edges were formed on the surface of SOS substrates by photolithography. SU-8 was spincoated onto the monolayer modified substrate at $800 \mathrm{rpm}$ for $30 \mathrm{~s}$ and then $3000 \mathrm{rpm}$ for 30 s. After spin-coating, the films were baked at $95^{\circ} \mathrm{C}$ for $2 \mathrm{~min}$ on a hot plate. The films were exposed to UV light through a mask then baked at $95^{\circ} \mathrm{C}$ for another $2 \mathrm{~min}$. The substrates were developed in EC solvent for $20 \mathrm{~s}$.

\subsection{Microcontact printing $(\mu C P)$}

PAH labelled with RBITC dye was prepared following a procedure described in [26]. The master was prepared following a previously reported protocol [27]. PDMS stamps were prepared by using a 10:1 ratio (v/w) of PDMS resin and curing agent (184 silicone elastomer, Sylgard). After degassing under vacuum, the PDMS was poured over a prepared silicon master and cured at $50^{\circ} \mathrm{C}$ overnight. Subsequently, the cured PDMS was peeled off from the master, treated in an oxygen plasma (pressure: 0.8 Torr, generator: $40 \mathrm{kHz} / 100 \mathrm{~W}$ ) for $30 \mathrm{~s}$ and then cut into $7 \mathrm{~mm} \times 7 \mathrm{~mm}$ pieces. The pattern of the PDMS stamp consisted of circular islands with a diameter of $40 \mu \mathrm{m}$ and $30 \mu \mathrm{m}$ gaps. Scheme $1 \mathrm{~b}$ illustrates the process of printing $\mathrm{PAH}$ on an undecylenic acid monolayer modified SOS substrate. The PDMS stamp was dipped in a $5 \mathrm{mg} / \mathrm{ml}$ RBITC-PAH solution for $40 \mathrm{~min}$. The soaked stamp was dried under nitrogen gas and placed onto the substrate for 5 min at room temperature. After removing the stamp, the patterned substrate was rinsed thoroughly with ultrapure (Milli-Q) water to remove unbound or loosely bound PAH. Atomic Force Microscopy (AFM, Dimension Icon, Bruker, US) in tapping mode was used to image the PAH pattern. A NCHVA (Silicon, 40N/m, Al reflective coating, Bruker) tip was used for scanning.

After LAPS measurements of the PAH pattern, the sample was coated with dsDNA by incubating with $24 \mu \mathrm{L}$ of TBA $(10 \mu \mathrm{M})$ and rTBA $(10 \mu \mathrm{M})$ in PBS buffer at pH 7.4 for 30 min. The presence of dsDNA was detected by the GeneFinder ${ }^{\mathrm{TM}}$ dye, which binds specifically to 
dsDNA. All fluorescence images were obtained using the Leica Epifluorescence Microscope DMI 4000B.

(a)

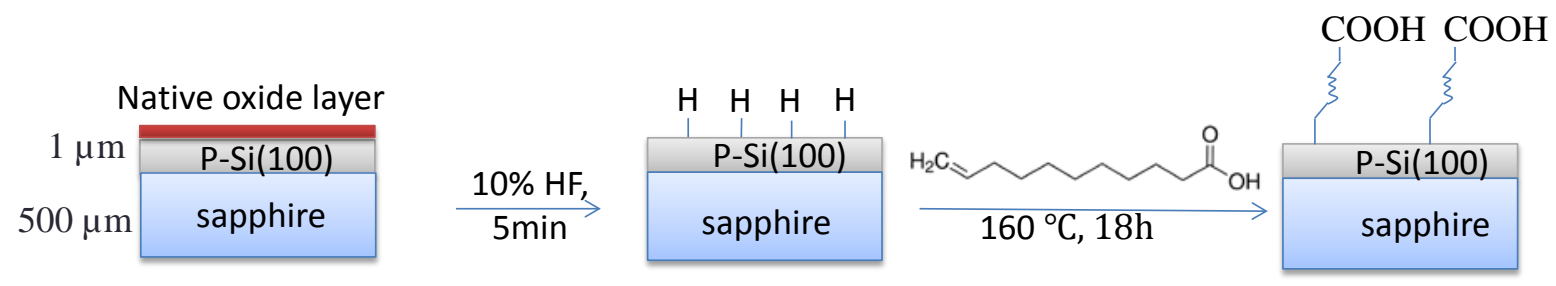

(b)

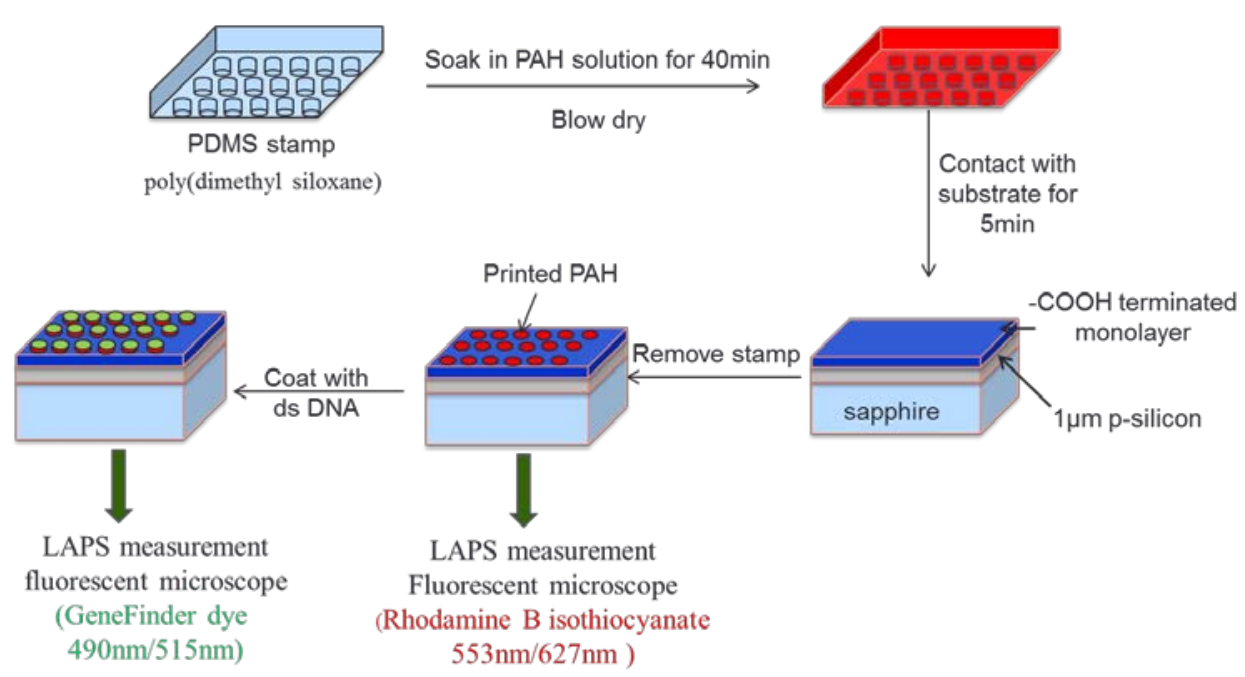

Scheme 1 (a) Schedule of undecylenic acid monolayer modification and (b) the stamping process of PAH dots and subsequent DNA adsorption.

\subsection{LAPS set-up}

The experimental setup for photocurrent measurements was described elsewhere [11]. LAPS/SPIM measurement were carried out using the same laser wavelengths as described previously, $405 \mathrm{~nm}, 633 \mathrm{~nm}, \lambda=1064 \mathrm{~nm}$, and $1250 \mathrm{~nm}$. The $405 \mathrm{~nm}$ laser was modulated electronically. All other lasers were modulated using a chopper. The modulation frequency was $1 \mathrm{kHz}$ for all photocurrent measurements. AC photocurrents excited by the modulated light were measured using an EG\&G 7260 lock-in amplifier.

The leakage current for undecylenic acid modified SOS was measured at room temperature using an Autolab PGSTAT 30. All potentials were measured vs. an $\mathrm{Ag} / \mathrm{AgCl}(3 \mathrm{M} \mathrm{KCl})$ electrode.

\section{Results and discussion}

\subsection{Characterisation of the undecylenic acid modified SOS substrates}

As both, 1-octadecene and undecylenic acid were expected to react with the $\mathrm{H}$-terminated surface in a similar manner and undecylenic acid was considered more useful for further modification, only the undecylenic acid modified surfaces were investigated with XPS. The 
XPS spectra of the modified SOS surface (Figure 1a) showed the presence of $\mathrm{Si}, \mathrm{C}$, and $\mathrm{O}$, indicating the successful modification of the silicon substrate. The narrow scan spectrum of the $C$ 1s region included a main $C-C$ peak $(-283 \mathrm{eV})$ and a small $C=O$ peak $(\sim 288 \mathrm{eV})$ (Figure $1 \mathrm{~b}$ ). The binding energies observed were in good agreement with results reported elsewhere [24, 28]. A small oxidized silicon signal centred at $102 \mathrm{eV}$ was observed from the high-resolution Si 2p scan (Figure 1c) indicating that the monolayer had some defects with residual oxide remaining on the surface due to the ingress of air to the SOS substrate.
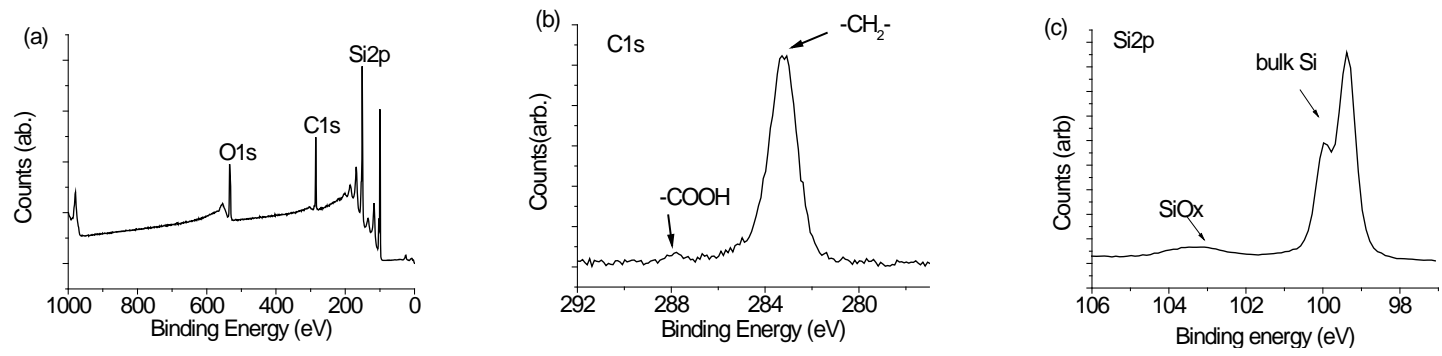

Figure 1. (a) XPS surveys scan of undecylenic acid monolayer modified SOS. High resolution scans for (b) carbon and (c) silicon.

Photocurrent measurements using the undecylenic acid modified SOS substrates displayed the expected behaviour for good quality LAPS substrates. The photocurrent curves (Figure 2a) were steep allowing high-sensitivity LAPS measurements. The photocurrent curves were shown to be stable for at least $5 \mathrm{~h}$ in buffer. The suitability of the organic monolayers as an insulator was further confirmed by the low leakage current density of $\sim 0.7 \mu \mathrm{A} / \mathrm{cm}^{2}$ measured at an inversion voltage of $0.8 \mathrm{~V}$ (Figure 2(a)). The pH sensitivity of the carboxyl terminated monolayer modified surface was determined by LAPS. Figure 2 shows the normalized photocurrent-voltage (I-V) curves (b) measured in a series of $\mathrm{pH}$ buffer solutions and the corresponding calibration plot (c). At low pH (3-4) and at high pH (8.5-9.4), no surface potential shift was detected, while in the $\mathrm{pH}$ range of 4-8.5, a linear relationship between voltage and $\mathrm{pH}$ was observed with a sensitivity of $30 \mathrm{mV} / \mathrm{pH}$. Considering that the $\mathrm{p} K_{a}$ of undecylenic acid is 4.5 , the modified silicon surface shows a pH sensitivity over a greater $\mathrm{pH}$ range than expected. It is assumed that some of the $\mathrm{pH}$ sensitivity can be attributed to the presence of silicon dioxide found on the surface (see XPS results above). The $\mathrm{pH}$ sensitivity of $30 \mathrm{mV} / \mathrm{pH}$ is similar to the results reported for $\mathrm{SiO}_{2}$ films [29, 30]. 

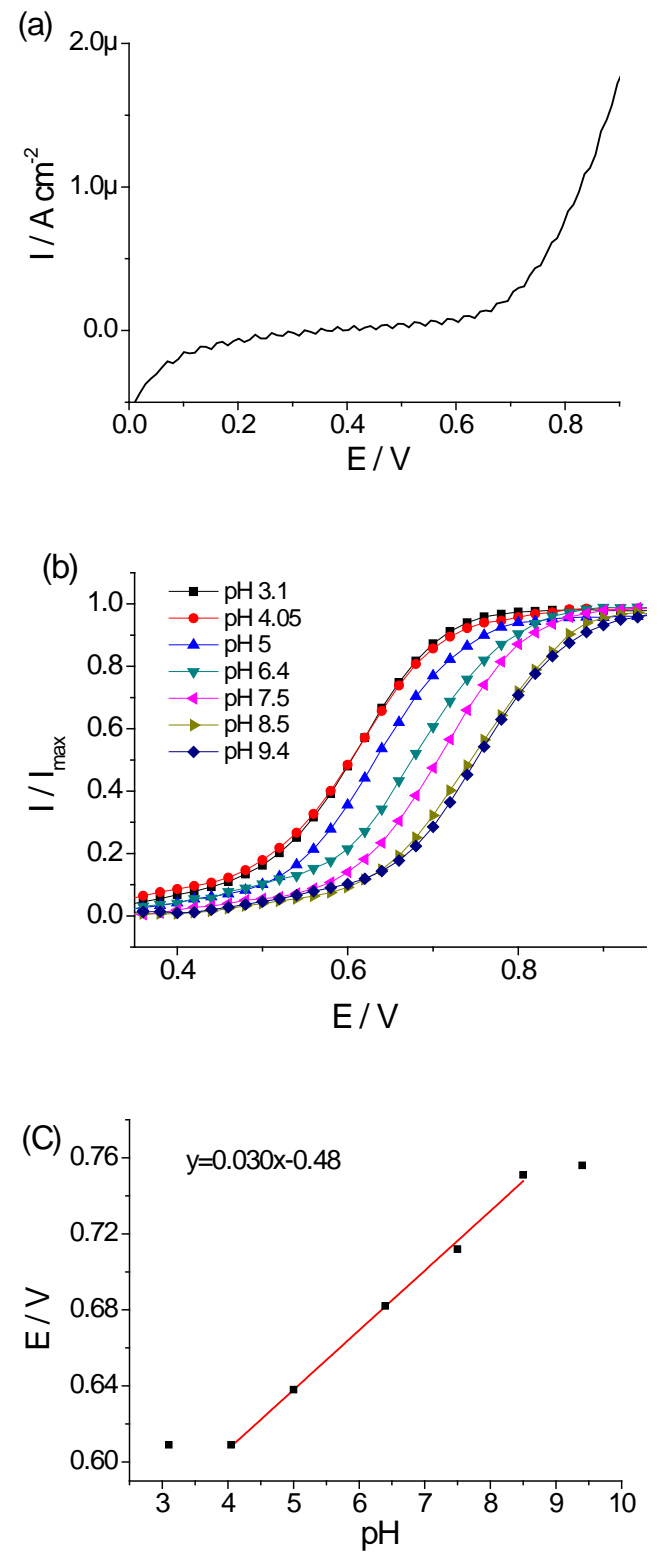

Figure 2 (a) Leakage current-voltage curves for undecylenic acid monolayer modified SOS. Sweep rate: $0.01 \mathrm{~V} / \mathrm{s}$ (b) Normalized I-V curves of a LAPS structure based on $-\mathrm{COOH}$ terminated monolayer $\left(I_{\max }=49 \pm 1.6 \mathrm{nA}\right)$; corresponding calibration plot $(c)$ for a series of different $\mathrm{pH}$ values.

\subsection{Resolution measurements}

As shown in [11], good lateral resolution of photocurrent measurements can be achieved using a SOS substrate with an anodic oxide as the insulator. In this work, we investigated the possibility of achieving similar results using a considerably thinner insulator consisting only of an organic monolayer. The resolution was measured by scanning a focused laser beam from an uncoated to an SU-8 coated part of the sample using back illumination while biasing the sample towards inversion (see inset in Figure 3a). Measuring in inversion means that the photocurrent contrast between coated and uncoated parts of the sample is caused entirely by the difference in impedance of the monolayer and the SU-8 coated monolayer resulting in a SPIM measurement [7]. The sharp edge of the photoresist allowed precise 
evaluation of the resolution. The photocurrent line scans for SOS substrates modified with an octadecyl monolayer at different laser wavelengths are shown in Figure $3 \mathrm{a}$. The results clearly show that the resolution obtained using a single photon effect can be improved by reducing the laser wavelength since the focus is wavelength dependent. However, even at a wavelength as short as $405 \mathrm{~nm}$, the resolution was limited to $1.6 \mu \mathrm{m}$, presumably due to stray light and multiple reflections in the sapphire substrate, which can also cause a photocurrent. These effects can be reduced using a two-photon effect. The substrate modified with an octadecyl monolayer showed sub-micrometre resolution at a wavelength of $1250 \mathrm{~nm}$, which has energy smaller than the band gap of silicon and can induce a twophoton effect as shown previously [11]. The resolution obtained at different wavelengths is comparable to the resolution measured previously using an SOS substrate with an anodic oxide [11]).

A marked difference was seen when the photocurrent contrast between coated and uncoated areas was compared. The photocurrent ratio for uncoated and coated areas at a wavelength of $405 \mathrm{~nm}$ was 12 for the monolayer modified samples but only 3.0 for the anodic oxide modified samples used previously [11]. This increase in the photocurrent contrast is due to the significantly smaller impedance of the ultrathin organic monolayers compared to the anodic oxide and clearly demonstrates the potential of increasing the sensitivity of LAPS and SPIM when employing an organic monolayer as the insulator rather than conventional insulators. To verify that the improvement in the photocurrent contrast was indeed due to the difference in impedance of the insulator, capacitance-voltage measurements for SOS substrates with both types of insulator were carried out at $1 \mathrm{kHz}$. The accumulation capacitance for samples with anodic oxide $(0.312 \mu \mathrm{F} \mathrm{cm}-2)$ was found to be 5.3 times smaller than the accumulation capacitance of samples with an organic monolayer $\left(1.65 \mu \mathrm{F} \mathrm{cm}{ }^{-2}\right)$. This factor was somewhat larger than expected from the difference in photocurrent contrast. Other quantities to be considered would be the electrolyte resistance and the capacitance of the space charge layer in the semiconductor. The serial resistance due to the electrolyte $\left(R_{e l}\right)$ for an illuminated area of $1.5 \mu \mathrm{m}$ in diameter can be estimated analogous to microelectrodes of the same size using equation 1 derived for a cell with spherical symmetry [31]:

$R_{e l}=\frac{1}{4 \pi \kappa r}\left(1-\frac{r}{L}\right)$

where $K$ is the conductivity of the electrolyte, $r$ the radius of the electrode and $L$ the distance between working and reference electrodes. The electrolyte resistance can be estimated to $76 \mathrm{k} \Omega$, which is negligible compared to the impedance of the anodic oxide ( $29 \mathrm{G} \Omega$ ) or organic monolayer $(5.5 \mathrm{G} \Omega$ ) in the illuminated area. It is therefore assumed that the discrepancy was due to the fact that the capacitance of the monolayer was measured in accumulation, while photocurrent measurements were carried out while biasing the sample towards inversion. 

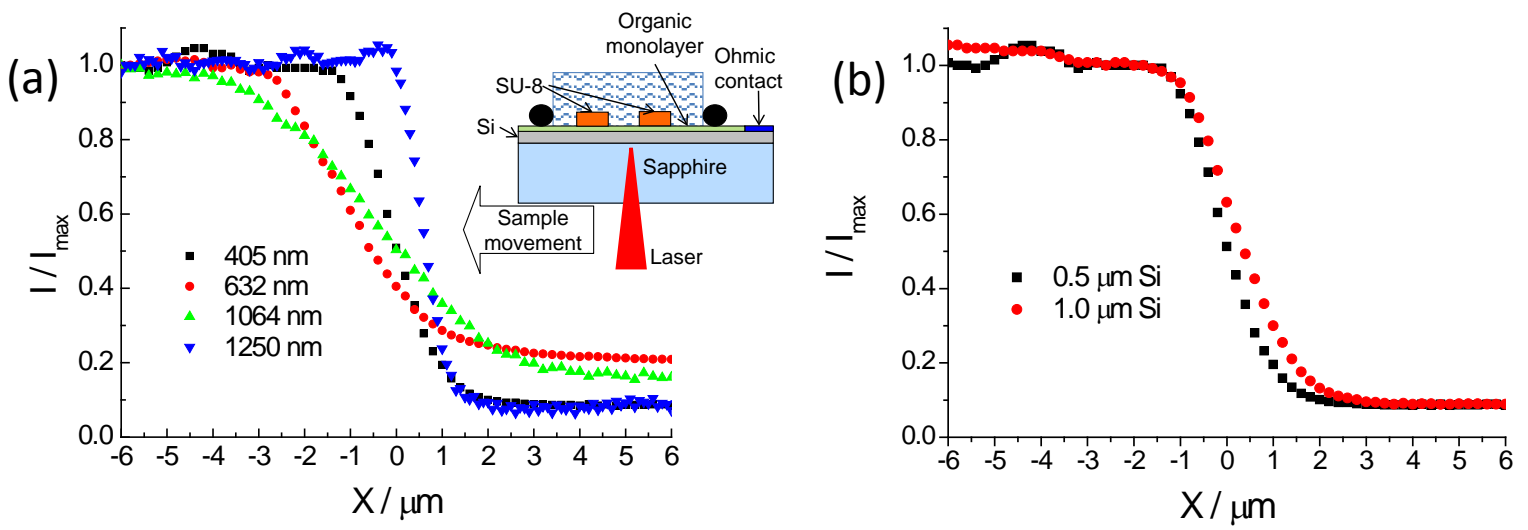

Figure 3 Normalised photocurrent line scans across SU-8 edge (a) at different laser wavelengths using SOS substrate with 1-octadecene grafted onto the silicon as the insulator and a silicon thickness of $0.5 \mu \mathrm{m}$; estimated resolution (the distance required to achieve a photocurrent drop to 1/e of the total drop): $1.6 \mu \mathrm{m}$ at $405 \mathrm{~nm}\left(I_{\max }=12 \mathrm{nA}\right), 2.2 \mu \mathrm{m}$ at 632 $\mathrm{nm}\left(I_{\max }=27 \mathrm{nA}\right), 3.6 \mu \mathrm{m}$ at $1064 \mathrm{~nm}\left(I_{\max }=60 \mathrm{nA}\right)$ and $0.8 \mu \mathrm{m}$ at $1250 \mathrm{~nm}\left(I_{\max }=30 \mathrm{nA}\right)$; inset shows sample structure; (b) at $405 \mathrm{~nm}$ using SOS substrate with undecylenic acid grafted onto silicon layers with different thickness; estimated resolution: $1.6 \mu \mathrm{m}$ for $0.5 \mu \mathrm{m}$ thick silicon and $2.0 \mu \mathrm{m}$ for $1 \mu \mathrm{m}$ thick silicon

We also investigated the influence of the type of organic monolayer and the thickness of the silicon on the resolution. Using undecylenic acid is attractive as it offers the option of functionalising the surface for different sensor and imaging applications. Photocurrent line scans across an SU-8 edge for SOS modified with undecylenic acid for two different silicon layer thicknesses are presented in Figure 3b. Undecylenic acid modified SOS with a $0.5 \mu \mathrm{m}$ thick silicon layer showed the same resolution $(1.6 \mu \mathrm{m})$ as the 1-octadecene modified samples with the same silicon thickness. Using SOS with a $1 \mu \mathrm{m}$ thick silicon layer resulted in a somewhat worse resolution of $2.0 \mu \mathrm{m}$. However, using $1 \mu \mathrm{m}$ thick silicon in conjunction with a laser wavelength of $405 \mathrm{~nm}$ has the advantage that all the light is absorbed by the silicon layer excluding any interference of the light with any biological sample or pattern deposited onto the front side of the sensor. It was therefore decided to carry out further investigations using undecylenic acid modified SOS substrates with a $1 \mu \mathrm{m}$ thick silicon layer and a laser wavelength of $405 \mathrm{~nm}$ for LAPS imaging.

\subsection{LAPS imaging with undecylenic acid modified SOS substrates}

Both, XPS and resolution measurements, showed that undecylenic acid monolayers can successfully be introduced as an insulator in LAPS. To validate surface potential imaging by LAPS, a RBITC-PAH pattern was printed onto the substrate. Figures $4 a$ and $b$ show the fluorescent microscope image of the RBITC-PAH pattern and the corresponding LAPS image measured in depletion at $0.488 \mathrm{~V}$. The fluorescent image demonstrated a good agreement with the LAPS image of the polyelectrolyte pattern. At the chosen voltage, the photocurrent was greater on the PAH pattern (bright islands shown in Figure 4b) than on the bare monolayer surface. This corresponds to the positive charge introduced by the polycationic $\mathrm{PAH}$.

Incubating the PAH patterned substrate with dsDNA solution is a simple and inexpensive way to generate a DNA microarray [32]. PAH-DNA binding is due to electrostatic attraction as DNA carries a negative charge. Figure $4 c$ presents the fluorescent microscope image of 
the Gene Finder ${ }^{\mathrm{TM}}$ labelled dsDNA array coated on PAH, which demonstrates a selective deposition of DNA. Figure 4d shows the LAPS image of the DNA array measured at $0.488 \mathrm{~V}$. Even though the pattern was still visible, the contrast between coated and uncoated areas decreased compared to the LAPS image before DNA coating (shown in Figure 4b). Due to the adsorption of the negatively charged DNA, the surface charge of the coated area became more similar to the surface charge of the undecylenic acid monolayer, which also carried a negative charge. Photocurrent curves for the areas on the pattern and on the undecylenic acid monolayer before and after DNA adsorption are shown in Figures $4 \mathrm{e}$ and $4 \mathrm{f}$. Before dsDNA coating, the lower part of the photocurrent curve on the PAH pattern shifted by $-41 \mathrm{mV}$ compared to the bare undecylenic acid modified surface causing a step to appear in the curve. After dsDNA adsorption, the lower part of the I-V curve shifted by $+20 \mathrm{mV}$. This shows that our LAPS devices are capable of measuring surface charge effects with spatial resolution. Adsorbing positively charged $\mathrm{PAH}$ to the negatively charged monolayer at $\mathrm{pH} 7.4$ resulted in a shift of the I-V curve to negative values. However, after incubating with negatively charged DNA, a negative surface charge was introduced resulting in a positive shift of the I-V curve. The directions and orders of magnitude of the potential shifts due to $\mathrm{PAH}$ and DNA adsorption are consistent with the result measured by Poghossian et al. [30, 33] using capacitance-voltage measurements at $\mathrm{p}-\mathrm{Si}-\mathrm{SiO}_{2}-\mathrm{Ta}_{2} \mathrm{O}_{5}$ and $\mathrm{p}-\mathrm{Si}-\mathrm{SiO}_{2} \mathrm{EIS}$ sensors. 
(a)

(c)
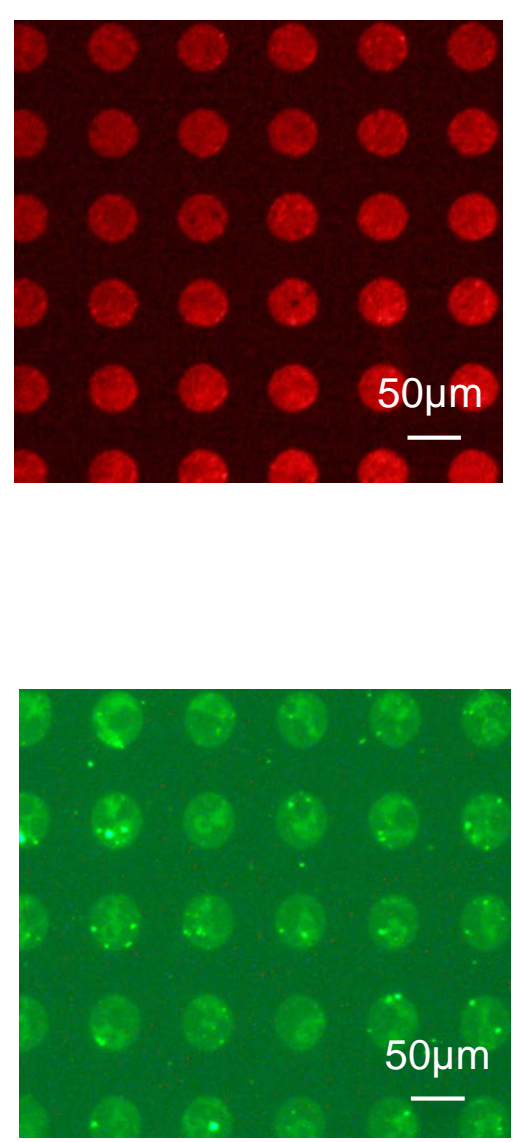

(e)

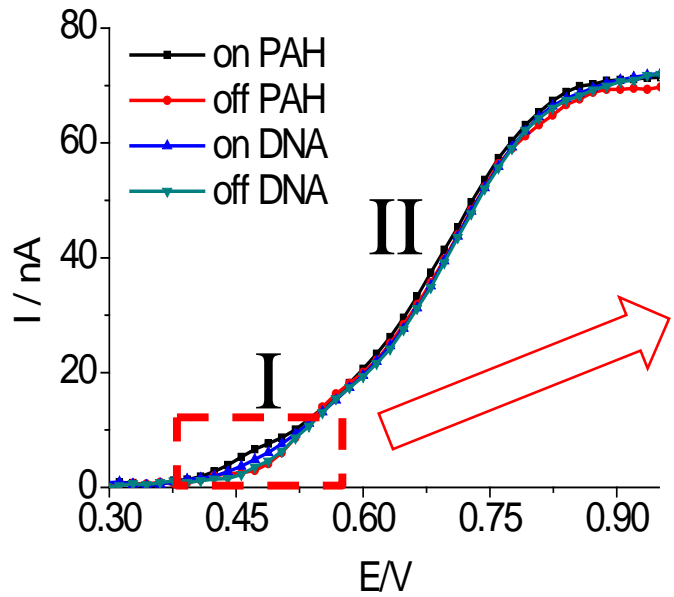

(b)

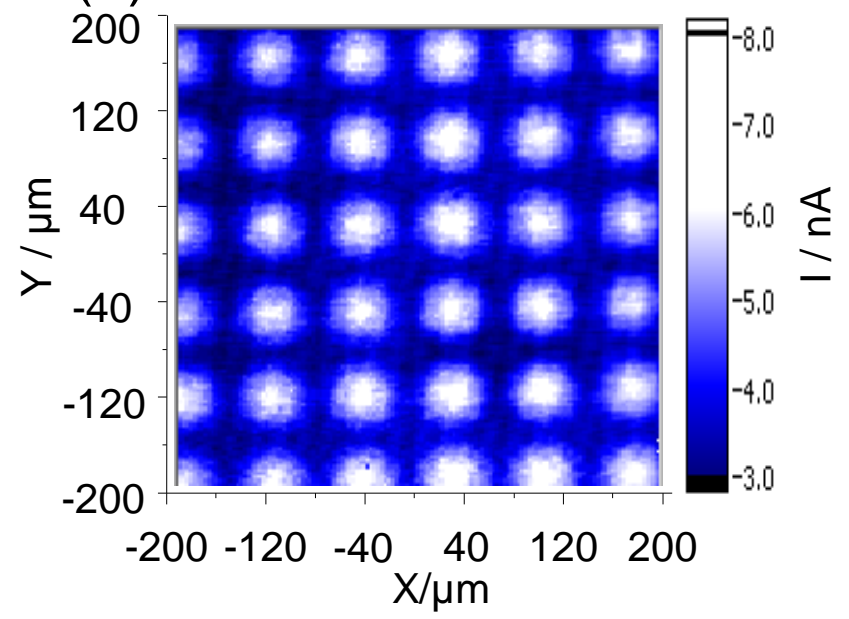

(d)

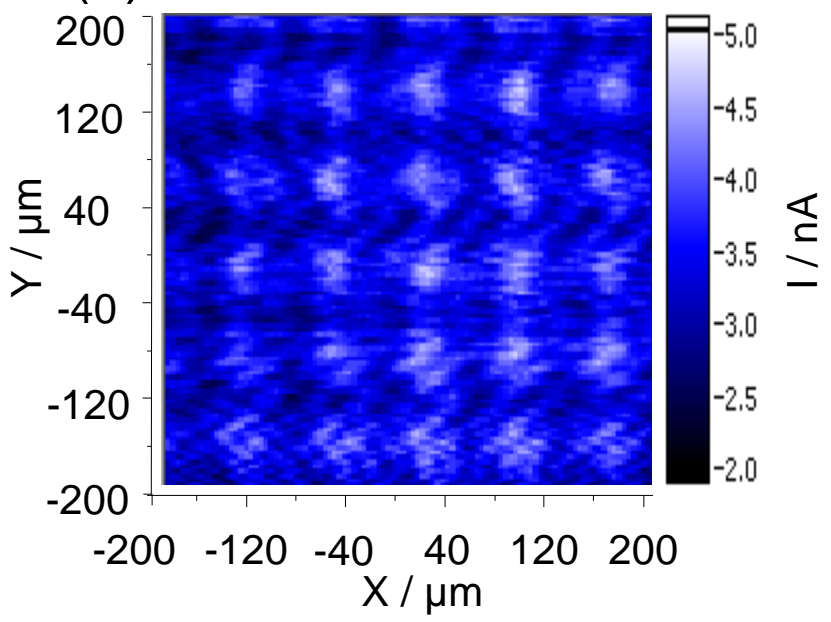

(f)

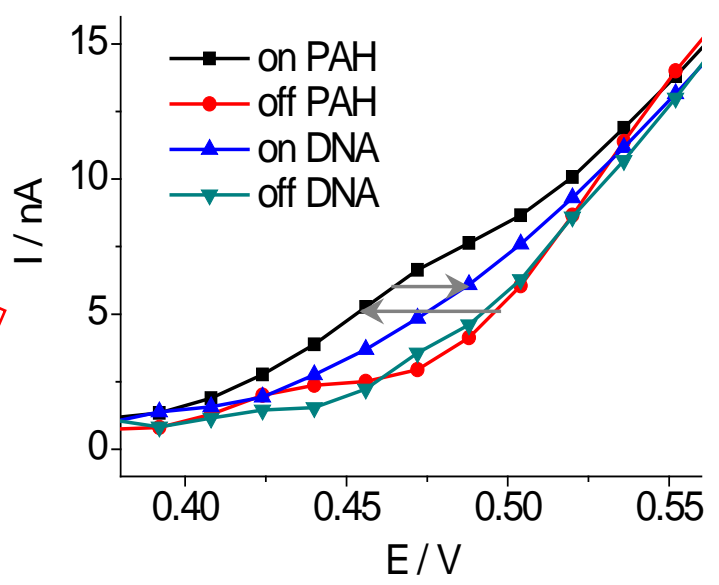

Figure 4. RBITC-PAH dots printed on an undecylenic acid SAM (a) fluorescent micrograph (b) corresponding LAPS image; Gene Finder labelled dsDNA adsorbed on PAH dots (c) fluorescence micrograph (d) corresponding LAPS image; LAPS images (b) and (d) measured at $0.488 \mathrm{~V}$. (e) I-V curves for PAH and PAH/DNA patterned on undecylenic acid modified SOS substrate and (f) zoomed graph in the depletion region. 
When the laser was focused onto the PAH coated part of the sample, a step appeared in the photocurrent curve (Figure 4e, part I) as the potential shift due to the change in surface charge appeared only in the lower part (I) of the I-V curves. This indicates that within the illuminated part of the sample, areas with different surface charges exist. This behaviour is consistent with incomplete coverage with $\mathrm{PAH}$ due to the printing process and is analogous to steps in capacitance voltage curves found in electrolyte/LaF $3 /$ silicon structures due to defects in the $\mathrm{LaF}_{3}$ film [34]. Some of the surface was coated with PAH exhibiting a positive surface charge, while other parts of the surface remained negatively charged due to the undecylenic acid monolayer. Part I of the I-V curves was clearly affected by charge of the $\mathrm{PAH}$ or PAH/DNA, while part II of the I-V curves remained unaffected displaying the behaviour of the bare undecylenic acid surface within the pattern. AFM images of a PAH dot confirmed the incomplete adsorption of PAH onto the substrate (Figure 5). In some parts of the PAH dot, the undecylenic acid monolayer was exposed. The average thickness of the $\mathrm{PAH}$ layer estimated from the AFM data was about 4.5-5 nm.
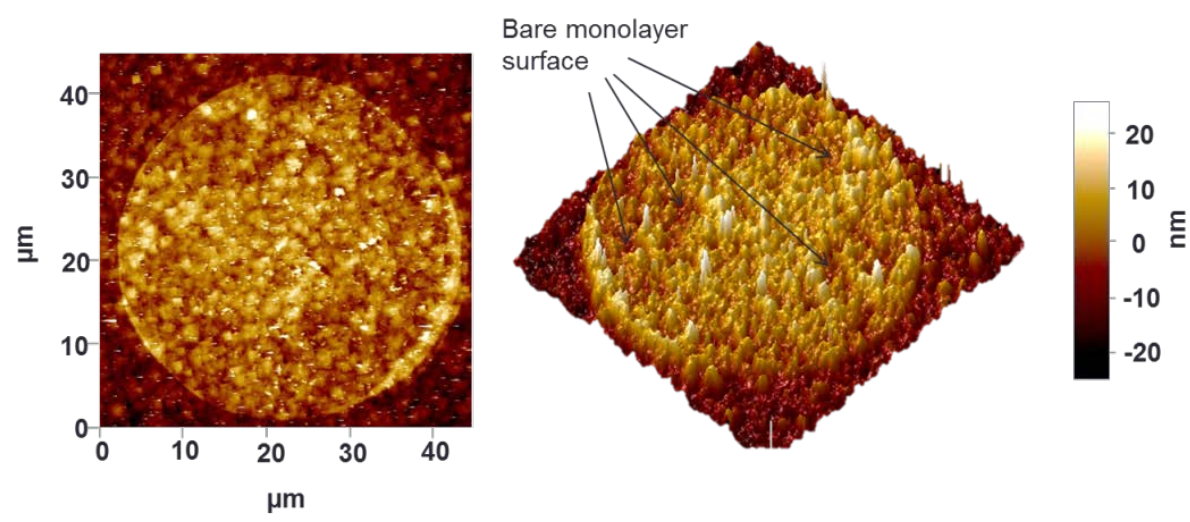

Figure 5. AFM analysis of patterned PAH coated on SAMs modified SOS substrate.

\section{Conclusions}

Good sensitivity and high spatial resolution of LAPS have been obtained using SOS substrates modified with 1-octadecene or undecylenic acid monolayers. The resolution was shown to be independent of the organic monolayer used. This is the first time that a selfassembled monolayer has been used as the insulator in LAPS measurements, which will broaden the applicability of LAPS by providing reproducible, ultrathin insulators that allow LAPS and SPIM measurements with high sensitivity and by introducing a controlled way for surface functionalization. A PAH pattern on the undecylenic acid monolayer modified SOS substrate was prepared using microcontact printing. DNA adsorption on the PAH template pattern was achieved purely by electrostatic interaction. LAPS images for PAH and PAH/DNA patterns were obtained, which were in good agreement with the corresponding fluorescent microscope images. The voltage shifts in the depletion region of the I-V curves indicate a good sensitivity for the measurement of the surface charge effects induced by the adsorption of PAH and DNA. Incomplete coverage with PAH was deduced from the partial shifts in the depletion region of the I-V curves and confirmed by AFM images. This indicates that LAPS may be able to determine the coverage of charged polymers, DNA or other biomolecules. 


\section{Acknowledgements}

The authors are grateful to the China Scholarship Council for funding, the EU for providing a Marie-Curie International Incoming Fellowship (MIF1-CT-2005-007808) and travel funds (PIRSES-GA-2009-247641), NEXUS (UK) for XPS measurements, Jean-Noël Chazalviel (École Polytechnique, CNRS, Palaiseau, France) for help with the monolayer modification of silicon, Matteo Palma's Research Group (Queen Mary University of London) for AFM measurements, Burcu Colak for help with the PDMS stamp preparation and Gleb Sukhorukov's group for help with the PAH labelling.

\section{References}

[1] J. Suzurikawa, M. Nakao, R. Kanzaki, H. Takahashi, Microscale pH gradient generation by electrolysis on a light-addressable planar electrode, Sensors and Actuators B: Chemical, 149(2010) 205-11.

[2] Y.G. Mourzina, Y.E. Ermolenko, T. Yoshinobu, Y. Vlasov, H. Iwasaki, M.J. Schöning, Anion-selective light-addressable potentiometric sensors (LAPS) for the determination of nitrate and sulphate ions, Sensors and Actuators B: Chemical, 91(2003) 32-8.

[3] K.-i. Miyamoto, T. Wagner, T. Yoshinobu, S.i. Kanoh, M.J. Schöning, Phase-mode LAPS and its application to chemical imaging, Sensors and Actuators B: Chemical, 154(2011) 2832.

[4] T. Wagner, M.J. Schöning, Light-addressable potentiometric sensors (LAPS): recent trends and applications, Comprehensive Analytical Chemistry, 49(2007) 87-128.

[5] D.G. Hafeman, J.W. Parce, H.M. McConnell, Light-addressable potentiometric sensor for biochemical systems, Science, 240(1988) 1182-5.

[6] B. Stein, M. George, H.E. Gaub, W.J. Parak, Extracellular measurements of averaged ionic currents with the light-addressable potentiometric sensor (LAPS), Sens Actuator BChem, 98(2004) 299-304.

[7] S. Krause, W. Moritz, H. Talabani, M. Xu, A. Sabot, G. Ensell, Scanning Photo-Induced Impedance Microscopy - Resolution studies and polymer characterization, Electrochim Acta, 51(2006) 1423-30.

[8] P.H. Chen, X.D. Liu, B.Q. Wang, G. Cheng, P. Wang, A biomimetic taste receptor cellbased biosensor for electrophysiology recording and acidic sensation, Sens Actuator BChem, 139(2009) 576-83.

[9] L.P. Du, C.S. Wu, H. Peng, L.H. Zhao, L.Q. Huang, P. Wang, Bioengineered olfactory sensory neuron-based biosensor for specific odorant detection, Biosens Bioelectron, 40(2013) 401-6.

[10] N. Hu, C.X. Wu, D. Ha, T.X. Wang, Q.J. Liu, P. Wang, A novel microphysiometer based on high sensitivity LAPS and microfluidic system for cellular metabolism study and rapid drug screening, Biosens Bioelectron, 40(2013) 167-73.

[11] L. Chen, Y. Zhou, S. Jiang, J. Kunze, P. Schmuki, S. Krause, High resolution LAPS and SPIM, Electrochemistry Communications, 12(2010) 758-60.

[12] Y. Nakato, S. Tonomura, H. Tsubomura, The Catalytic Effect of Electrodeposited Metals on the Photo-Reduction of Water at p-type Semiconductors, Berichte der

Bunsengesellschaft für physikalische Chemie, 80(1976) 1289-93.

[13] E. Mahé, F. Rouelle, I. Darolles, D. Devilliers, Electrochemical characterization of silicon electrodes: Part 1: Capacitance-Voltage Method, Journal of New Materials for Electrochemical Systems, 9(2006) 257.

[14] H. Föll, Properties of silicon-electrolyte junctions and their application to silicon characterization, Applied Physics A, 53(1991) 8-19.

[15] M. George, W.J. Parak, I. Gerhardt, W. Moritz, F. Kaesen, H. Geiger, et al., Investigation of the spatial resolution of the light- addressable potentiometric sensor, Sens Actuator A-Phys, 86(2000) 187-96. 
[16] Y. Liu, S. Yamazaki, S. Yamabe, Y. Nakato, A mild and efficient Si (111) surface modification via hydrosilylation of activated alkynes, Journal of Materials Chemistry, 15(2005) 4906-13.

[17] H. Asanuma, E.M. Bishop, H.-Z. Yu, Electrochemical impedance and solid-state electrical characterization of silicon (1 111 ) modified with $\omega$-functionalized alkyl monolayers, Electrochimica Acta, 52(2007) 2913-9.

[18] A. Vilan, O. Yaffe, A. Biller, A. Salomon, A. Kahn, D. Cahen, Molecules on Si: Electronics with Chemistry, Advanced Materials, 22(2010) 140-59.

[19] S. Ciampi, J.B. Harper, J.J. Gooding, Wet chemical routes to the assembly of organic monolayers on silicon surfaces via the formation of Si-C bonds: surface preparation, passivation and functionalization, Chemical Society Reviews, 39(2010) 2158-83.

[20] L. Touahir, P. Allongue, D. Aureau, R. Boukherroub, J.N. Chazalviel, E. Galopin, et al., Molecular monolayers on silicon as substrates for biosensors, Bioelectrochemistry, 80(2010) 17-25.

[21] G.-J. Zhang, M.J. Huang, J.A.J. Ang, E.T. Liu, K.V. Desai, Self-assembled monolayerassisted silicon nanowire biosensor for detection of protein-DNA interactions in nuclear extracts from breast cancer cell, Biosensors and Bioelectronics, 26(2011) 3233-9. [22] R. Voicu, R. Boukherroub, V. Bartzoka, T. Ward, J.T.C. Wojtyk, D.D.M. Wayner, Formation, Characterization, and Chemistry of Undecanoic Acid-Terminated Silicon Surfaces: Patterning and Immobilization of DNA, Langmuir, 20(2004) 11713-20. [23] A. Faucheux, A.C. Gouget-Laemmel, C. Henry de Villeneuve, R. Boukherroub, F. Ozanam, P. Allongue, et al., Well-Defined Carboxyl-Terminated Alkyl Monolayers Grafted onto H-Si(111): Packing Density from a Combined AFM and Quantitative IR Study, Langmuir, 22(2005) 153-62.

[24] M. Perring, S. Dutta, S. Arafat, M. Mitchell, P.J.A. Kenis, N.B. Bowden, Simple Methods for the Direct Assembly, Functionalization, and Patterning of Acid-Terminated Monolayers on Si(111), Langmuir, 21(2005) 10537-44.

[25] A. Faucheux, A.C. Gouget-Laemmel, C.H. de Villeneuve, R. Boukherroub, F. Ozanam, $\mathrm{P}$. Allongue, et al., Well-defined carboxyl-terminated alkyl monolayers grafted onto $\mathrm{H}-\mathrm{Si}(111)$ : Packing density from a combined AFM and quantitative IR study, Langmuir, 22(2006) 15362.

[26] Y. Qiangying, UV-triggered Encapsulation and Release by Multilayer Microcapsules: Queen Mary univeristy of London; 2013.

[27] J.E. Gautrot, B. Trappmann, F. Oceguera-Yanez, J. Connelly, X. He, F.M. Watt, et al., Exploiting the superior protein resistance of polymer brushes to control single cell adhesion and polarisation at the micron scale, Biomaterials, 31(2010) 5030-41.

[28] T. Strother, W. Cai, X. Zhao, R.J. Hamers, L.M. Smith, Synthesis and characterization of DNA-modified silicon (111) surfaces, Journal of the American Chemical Society, 122(2000) 1205-9.

[29] C. Cane, I. Gracia, A. Merlos, Microtechnologies for pH ISFET chemical sensors, Microelectronics Journal, 28(1997) 389-405.

[30] A. Poghossian, M.H. Abouzar, M. Sakkari, T. Kassab, Y. Han, S. Ingebrandt, et al., Field-effect sensors for monitoring the layer-by-layer adsorption of charged macromolecules, Sensors and Actuators B: Chemical, 118(2006) 163-70.

[31] O. Koster, W. Schuhmann, H. Vogt, W. Mokwa, Quality control of ultra-microelectrode arrays using cyclic voltammetry, electrochemical impedance spectroscopy and scanning electrochemical microscopy, Sens Actuator B-Chem, 76(2001) 573-81.

[32] Z. Wang, P. Zhang, B. Kirkland, Y. Liu, J. Guan, Microcontact printing of polyelectrolytes on PEG using an unmodified PDMS stamp for micropatterning nanoparticles, DNA, proteins and cells, Soft Matter, 8(2012) 7630-7.

[33] A. Poghossian, S. Ingebrandt, M. Abouzar, M. Schöning, Label-free detection of charged macromolecules by using a field-effect-based sensor platform: Experiments and possible mechanisms of signal generation, Applied Physics A, 87(2007) 517-24.

[34] J. Szeponik, W. Moritz, A NEW STRUCTURE FOR CHEMICAL SENSOR DEVICES, Sens Actuator B-Chem, 2(1990) 243-6. 